\title{
Tunnel Effect With Moving Tunnel*
}

\author{
Yoshie Kiritani \\ Chiba University, Chiba, Japan
}

\begin{abstract}
Tunnel effect is a perception of continuous but hidden movement under a screen. The present study confirmed the optimal interval of occlusion to see the effect, when both a moving object and the screen move together. Firstly, four criteria were confirmed to check the tunnel effect in the standard situation where the screen was standing. In experiment 1 , a small square (object) and vertically long rectangle (screen) moved in the same direction and passed each other. As a result, if the object moved faster than the screen, the optimal interval of occlusion was not so different from that in the standard one. However, if the screen moved faster than the object, the longer interval was needed. A kind of entraining effect was seen. Also, in experiment 2 where the object and the screen approached and passed each other, the similar tendency was confirmed. Moreover, if they moved with the same speed, the tunnel effect was difficultly seen.
\end{abstract}

Keywords: amodal completion, tunnel effect, motion perception

We can see object's movement, even if there is no physical movement. Animation films use our perceptual property and embody the movement. Nowadays, we are familiar with the movement in any media like moving icons in Website. Although designers empirically learn many things, it pays to efficiently know fundamental perceptual properties, when and how we see the movement, for instance. Our perceptual system has own rule that is not the same as the physical one, which is well-represented in geometrical illusion.

The present study provides the fundamental data of tunnel effect, a phenomenon of movement. Although only two experts participated in the experiments, the total number of judgment was not negligible. In experiment 1, there were 160 combinations of conditions. But, in each combination, 11 steps of a variable were judged and the total number of trials was computed by multiplying 160 by 11, although some trials were broken off according to the procedure of psychophysics. In experiment 2, the 240 combinations of conditions were multiplied by 11 , too. The number of participants was too small for a quantitative research. However, the author found a crucial issue during the preliminary experiment where six naïve observers had participated in. The naïve observers were indifferent to the aspect of the change of the phenomenon. They saw different aspects, accepted them and judged as the same aspect without any uncomfortable feelings. The point to know was the difference of these aspects. Thus, the experiments were conducted using experts of observation. As mentioned later, the purpose of research was not just a demonstration of these aspects of the phenomenon, but a measure of variables. In this sense, the small number of participants was an undeniable truth. Thus, the number of trials was large. The results will serve as a basis for initial trials of the tunnel effect with moving tunnel.

\footnotetext{
${ }^{*}$ This research was done under a grant from Canon Foundation in Europe in 2000.

Yoshie Kiritani, Ph.D., Department of Design Science, Graduate School of Engineering, Chiba University.
} 


\section{Definition of Problem}

When a small object is moving and its trajectory is hidden by a bigger object (see Figure 1), you can still see its continuous movement under a certain condition. If the masking interval is too small comparing to the standing screen, you will see two distinct objects moving on the sides of screen. If the interval is too large, you will see two moving objects or one object whose movement is under the screen vanishes. If the interval is perceptually proper to pass the screen, you can see continuous and existing movement of a single object despite of the screen. This is tunnel effect.

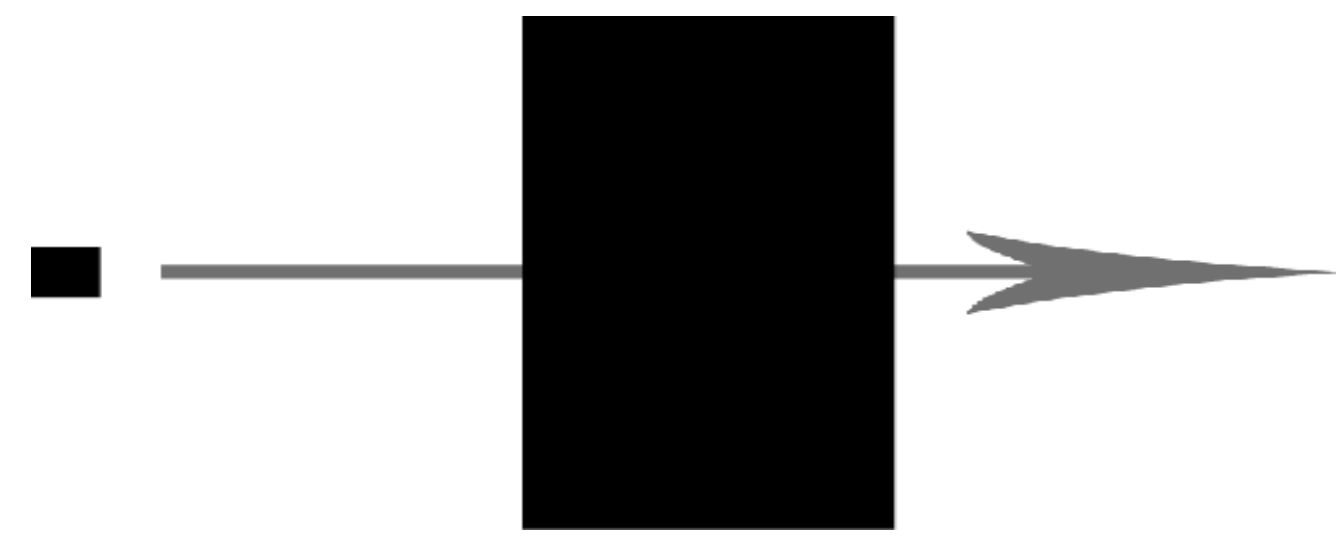

Figure 1. A situation of the traditional tunnel effect.

Firstly, described by Wundt (1902), von Kries (1902), Wertheimer (1912), the tunnel effect was investigated by Sampaio (1943) and Burke (1952). Perhaps, it was Wertheimer (1912) who gave a name to it, since he spoke of "Tunnelbeobachtung”. Surely, it was Linke (as cited in Wertheimer, 1912, pp. 224-225) who pointed out the fact that the movement under the screen is "unmittelbar gesehen (directly seen)". In other words, it seems that Linke foresaw the conceptual tool of "amodal presence", later developed by Michotte and Burke (1951) and Michotte, Thinès, and Crabbé (1964). There are some works of perceptual psychology to know the concept of amodal presence (Kanizsa, 1991; Metzger, 1975; Vezzani, 1998; Vicario \& Kiritani, 1999).

Here is a question about spatiotemporal conditions in the tunnel effect. To see it, certain interval is needed for the length of screen. Is this relationship between time and space always effective, if the screen also moves? In the tunnel effect in perceptual studies, the tunnel or the screen has not moved. If not only the small object but also the screen move together to keep spatiotemporal conditions for the tunnel effect, can we still see the effect? If the physical laws are powerful and effective in the perceptual field, the movement of screen has no influence on the tunnel effect. However, if the perceptual field has own laws, we will have another relationship between time and space according to which object moves in the situation. The present study confirms the optimal interval to see the tunnel effect in which two objects, the screen and the hidden object, move together.

However, the study met an obstacle. In the traditional tunnel effect, researchers have different opinions about the optimal interval. Sampaio (1943) and Burke (1952) said that the increase of interval must be less than the increase in the width of the screen to maintain the apparent continuity of the movement, and Brandt (1982) took the fact as a general rule that the optimal interval has to be in any case smaller than the physical occlusion interval, the time necessary for a real object that moves at a certain velocity to pass behind a real screen. However, Reynolds (1968) presented the opposite result, and moreover, Maruyama and Iwasaki (1973) ascertained differences in the optimal interval, expressed by a rather wide range of optimal intervals within the 
same observer, and considerable differences among observers.

The author examined a traditional experimental situation of the tunnel effect using six naïve observers to seek for the optimal interval. The width of screen and the speed of small moving object were systematically changed. The observers' task was to adjust the occlusion interval till they came to see the tunnel effect. They were instructed that it was a perception of total continuous movement including indirect movement under the screen and the continuity could also be represented by the reappearance of the object just in time. When, during experimental trials, the observers said "The interval is too short" or "The object comes out from the screen too early", the experimenter prolonged the interval by step of $50 \mathrm{~m}$. When the observers said "The interval is too long" or "The object comes out too late", the experimenter shortened the interval by step of $50 \mathrm{~m}$. There were seven starting values of the interval, which were presented in a randomized order. As a result, the adjusted temporal values went to be almost equal to the first randomized value given to each observer. Averaged number of adjustments is 0.9 times, which means that the observers hardly changed the interval to get the tunnel effect.

Moreover, there were some unexpected difficulties. Observers' verbal reports revealed that "the continuous movement" was not always equal to the "good timing of reappearance", although the experimenter explained them that these were the features of the tunnel effect. Especially, in the wider screen condition with slowest object's speed, the observers used timing strategies to check the existence of the tunnel effect. On the contrary, the strategy was abandoned and other strategy was adopted in narrow screen condition with highest objects' speed. Besides, it was found that the randomized presentation order heavily interfered with keeping up an assigned criterion, especially because of the sudden variations of speed. The naïve observers might smoothly switch the criterion and continuously report the tunnel effect. At least, a criterion was always satisfied.

Thus, it was concluded that experienced observers who were used to the psychological experiments should have checked the properties of the tunnel effect. Experiments in the present study needed a huge numbers of trials. It was difficult to find a patient person who continued to participate in the experiments. In the following experiments, the participants were just two persons, one of whom was the author. The purpose of the study was to confirm the optimal time interval to see the tunnel effect under the more dynamic situation. The consistent results reported later outweigh the negative of this study.

\section{Preliminary Experiment}

The purpose of the experiment was to confirm the difference of criteria of the tunnel effect. Here, the phenomenon is the traditional tunnel effect which would be seen with a standing screen. Observers were two experts. Experimental situations were displayed on a 19 monitor (Studio Works 995E, LG Electronics Inc.), $800 \times 600$ pixels resolution, $60 \mathrm{~Hz}$ refresh rate, controlled by a Macintosh PowerBook G3, operating with True Basic 2.72. A black square (hidden object: $6 \mathrm{~mm} \times 6 \mathrm{~mm}$ ) moved toward a black standing rectangle (screen) from left to right as shown in Figure 1. When the hidden object touched the screen, the former became smaller and finally merged. After a while, at the right side of the screen, another black square appeared and went ahead at the same speed before.

The height of the screen was $67.5 \mathrm{~mm}$ and its width was variable: $27,54 \mathrm{or} 81 \mathrm{~mm}$. The speed of the hidden object was 50,100 , or $200 \mathrm{~mm} / \mathrm{s}$. The interval of absence of square was also variable. Calculating the physically required interval to pass through the screen, there were 11 intervals to decrease or increase from it with each $15 \%:-75 \%,-60 \%,-45 \%,-30 \%$ and $-15 \%$ of physical interval, and physical interval itself, $+15 \%$, $+30 \%,+45 \%,+60 \%$ and $+75 \%$ of physical interval.

The observer's task was to judge if the experimental situation satisfied the following four criteria: (1) 
Continuity criterion was if the hidden object seemed to keep moving under the screen; (2) Timing criterion was if the hidden object seemed to emerge out from the screen after the proper masking interval; (3) Permanence criterion was if the hidden object seemed to still exist under the screen during the masking; and (4) Identity criterion was if the hidden object emerged from the right side of the screen was the same one as the first hidden object. These criteria were introduced because of the results of the former mentioned experiment and the description by Michotte and his colleagues (Michotte \& Burke, 1951; Michotte et al., 1964).

The method of limits was employed, since it favors a uniform and constant mode of observation. For each combination of screen width and object's speed, 11 intervals were presented in ascending series and in descending series. Both series were repeated for ten times.

Three widths of screen and three speeds of object made nine situations. The order of presentation of nine situations was randomized. The total number of trials that each participant took was 180 . In each trial, one of 11 intervals was presented in consecutive order and judged according to a certain criterion.

\begin{tabular}{|c|c|c|c|c|c|c|c|c|c|c|c|c|c|}
\hline & \multirow{2}{*}{$\begin{array}{c}\text { Width } \\
27\end{array}$} & \multirow{2}{*}{$\begin{array}{c}\text { Speed } \\
50\end{array}$} & \multicolumn{11}{|c|}{ Increass-decrease rate of interval } \\
\hline \multirow{3}{*}{ 袅 } & & & -75 & $-6 \mathrm{C}$ & -45 & -30 & -15 & $\mathrm{PI}$ & +15 & +30 & +45 & +50 & +75 \\
\hline & 27 & 100 & -75 & $-6 \mathrm{C}$ & -45 & -30 & -15 & $\mathrm{PI}$ & +15 & +30 & +45 & +60 & +75 \\
\hline & 27 & 200 & -75 & $-6 \mathrm{C}$ & -45 & -30 & -15 & $\mathrm{Pl}$ & +15 & +30 & +45 & +60 & +75 \\
\hline & 27 & 50 & -75 & $-6 \mathrm{C}$ & -45 & -30 & -15 & $\mathrm{PI}$ & +15 & +30 & +45 & +30 & +75 \\
\hline & 27 & 100 & -75 & $-6 C$ & -45 & -30 & -15 & $\mathrm{Pl}$ & +15 & +30 & +45 & +60 & +75 \\
\hline 竎 & 27 & 200 & -75 & $-6 \mathrm{C}$ & -45 & -30 & -15 & $\mathrm{PI}$ & +15 & +30 & +45 & +60 & +75 \\
\hline & 54 & 50 & -75 & -60 & -45 & -30 & -15 & PI & +15 & +30 & +45 & +60 & +75 \\
\hline & 54 & 100 & -75 & $-6 \mathrm{C}$ & -45 & -30 & -15 & $\mathrm{PI}$ & +15 & +30 & +45 & +60 & +75 \\
\hline & 27 & 100 & -75 & -60 & -45 & -30 & -15 & $\mathrm{Pl}$ & +15 & +30 & +45 & +50 & +75 \\
\hline 焉 & 27 & \begin{tabular}{|l|}
200 \\
\end{tabular} & -75 & -50 & -45 & -30 & -15 & $P 1$ & +15 & +30 & +45 & +50 & +75 \\
\hline 迹 & 54 & 200 & -75 & -50 & -45 & -30 & -15 & $P \mid$ & +15 & +30 & +45 & +60 & +75 \\
\hline & 27 & 50 & -75 & -60 & -45 & -30 & -15 & $\mathrm{PI}$ & +15 & +30 & +45 & +60 & +75 \\
\hline t5 & 27 & 100 & 75 & 60 & 45 & 30 & 15 & $P$ & +15 & +30 & +45 & +60 & +75 \\
\hline & 27 & \begin{tabular}{|l}
200 \\
\end{tabular} & -75 & -60 & -45 & -30 & -15 & $P \mid$ & +15 & +30 & +45 & +60 & +75 \\
\hline
\end{tabular}

Figure 2. Results of the preliminary experiment: "Width" and "speed" are conditions of the screen.

To avoid troublesome description of results, the best conditions for each criterion were just mentioned. The conditions of the width of screen, speed of object and increase-decrease ratio of interval in which each criterion satisfied over $80 \%$ of the time were presented in Figure 2. The width of screen should be smaller to see the tunnel effect, although the timing and the permanence were also satisfied in certain intervals of the 54 $\mathrm{mm}$ screen. For the continuity, the permanence and the identity, the preferred intervals depended on the speed of hidden object. If the speed became faster, the interval to satisfy the criterion was widened. At the speed of $200 \mathrm{~mm} / \mathrm{s}$, the preferred interval had wider range. On the other hand, the timing had rather narrow band in the 
preferred interval. Moreover, in the results appeared in Figure 2, if the standard was lowered to $70 \%$ satisfaction, the timing was also reported in the $200 \mathrm{~mm} / \mathrm{s}$ with $54 \mathrm{~mm}$ width and $100 \mathrm{~mm} / \mathrm{s}$ with $81 \mathrm{~mm}$ width.

Four criteria were features of the tunnel effect which appeared under the different conditions. The continuity and the identity were hardly satisfied, when the screen became wider. The difference between the continuity and the identity was represented in the favorable intervals; the continuity did not appear in longer intervals than the one physically required. The timing mostly showed constancy. The permanence would be hardly distinguished from the other criteria in the data. But, only this criterion was satisfied in the condition of $54 \mathrm{~mm}$ screen at the $200 \mathrm{~mm} / \mathrm{s}$ with $-30 \%$ intervals. In the following experiments, these four criteria were adopted to describe the tunnel effect, because they could uniquely define the tunnel effect.

\section{Experiment 1}

\section{Purpose}

The purpose was to confirm if the favorable masking intervals changed according to the movement of screen. In the experiment 1 , the hidden object and the screen moved in the same direction.

\section{Method}

Participants. Two observers in the preliminary experiment took part in the experiment. They had corrected-to-normal vision.

Apparatus and stimuli. The apparatus was the same as in the preliminary experiment. In the experimental situation, a black square (hidden object) moved from left to right, as in the preliminary experiment. But, in this experiment, a black rectangle (screen) also moved from left to right. When the hidden object moved faster than the screen, the hidden object stood to the left of the screen at first and after a while passed it (see Figure 3a). On the other hand, when the screen moved faster than the hidden object, the screen was at the left side of hidden object in the beginning (see Figure 3b). The faster moved at $200 \mathrm{~mm} / \mathrm{s}$ and the speed of the slower was $100 \mathrm{~mm} / \mathrm{s}$. Thus, in object-faster condition, the object moved at $200 \mathrm{~mm} / \mathrm{s}$ and the screen moved at $100 \mathrm{~mm} / \mathrm{s}$, and in screen-faster condition, the object moved at $100 \mathrm{~mm} / \mathrm{s}$ and the screen moved at $200 \mathrm{~mm} / \mathrm{s}$. Thus, the relative speed was always $100 \mathrm{~mm} / \mathrm{s}$. The hidden object was $6 \mathrm{~mm}$ on a side. The screen was $27 \mathrm{~mm}$ in width and $67.5 \mathrm{~mm}$ in height.

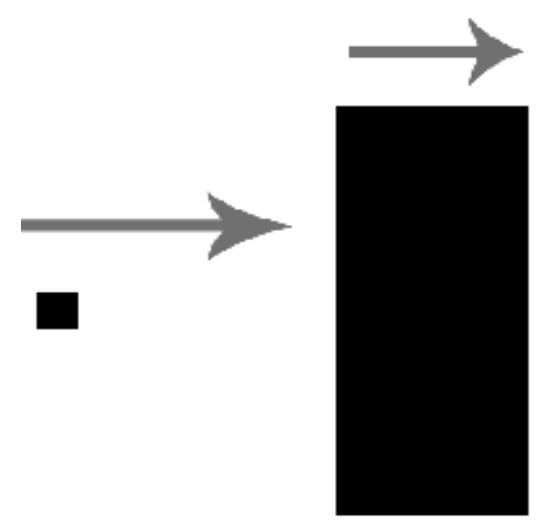

(a) Object-faster condition

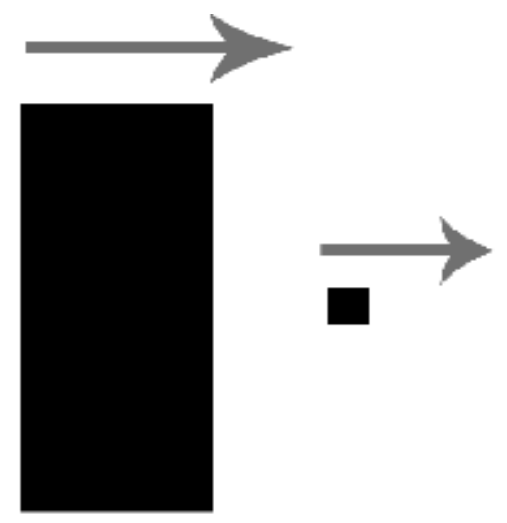

(b) Screen-faster condition

Figures 3. Starting position in the experiment 1. 
There were three experimental factors. The first factor was that four criteria described in the preliminary experiment were adopted. The second factor was which moved faster, the object or the screen. The third factor was 11 masking interval as in the preliminary experiment. Moreover, to increase trials, we introduced another size and speed conditions, which were not analyzed ${ }^{2}$.

Procedure. As in the preliminary experiment, the method of limits was employed. In each situation, 11 intervals were presented in ascending series and descending series. The observer's task was to judge if the experimental situation satisfied a criterion, that is, continuity, timing, permanence, or identity. Each situation was repeated in ten times.

\section{Results and Discussion}

Figure 4 shows averaged numbers of satisfied trials for four criteria as a function of the interval condition under the object-faster condition. Figure 5 is for the screen-faster condition. In the object-faster condition, the greatest frequencies of three out of four criteria appeared in minus area of the interval condition. On the other hand, in the screen-faster condition, most of the greatest frequencies appeared in plus area of the interval condition. The permanence criterion had a peak interval at the physical interval for both faster-object conditions.



Object Faster Condition

Figure 4. Averaged number of frequencies of four criteria as a function of the interval in the object-faster condition: for the continuity, $\square$ for the timing, $\boldsymbol{\Delta}$ for the permanence and $\circ$ for the identity.

\footnotetext{
${ }^{2}$ There were 6 conditions of object's speed (mm/s), screen's width (mm), and its speed (mm/s): (1) 100, 27, 50; (2) 50, 27, 100; (3) 200, 54, 100; (4) 100, 54, 200; (5) 400, 54, 200; and (6) 200, 54, 400.
} 


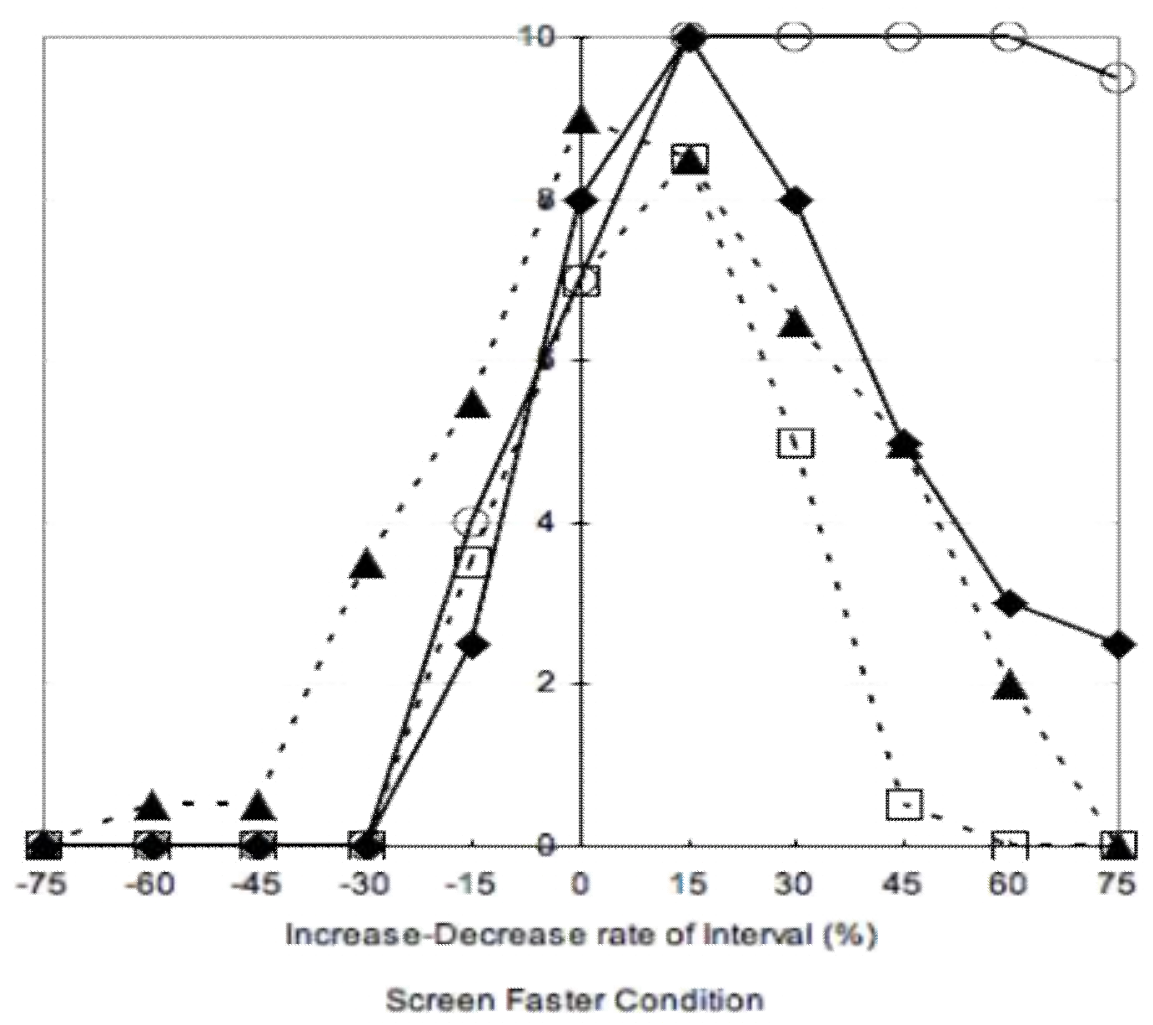

Figure 5. Averaged number of frequencies of four criteria as a function of the interval in the screen-faster condition:

$\checkmark$ for the continuity, $\square$ for the timing, $\boldsymbol{\Delta}$ for the permanence and $\circ$ for the identity.

A three-way ANOVA with repeated measure was conducted and significantly revealed a main effect of the criteria, the interval, an interaction between the criteria and the interval, between the faster-object and the interval and an interaction among three factors ${ }^{3}$. To know the difference of favorable interval for each criterion, we picked up the interaction among three factors. A simple-simple main effect revealed that for the continuity criterion and the timing criterion, the difference between the object-faster condition and the screen-faster condition was significant at the $-15 \%$ interval and at the $+15 \%$ interval. For the identity criterion, this difference in faster-object conditions was significant at the intervals of $-15 \%,+30 \%,+45 \%,+60 \%{ }^{4}$.

Thus, except the permanence condition, it was statistically shown that the difference of the faster-object conditions required different favorable intervals (seen Figures 4 and 5). The favorable intervals for the identity criterion in the screen-faster condition stand out, and the identity was continuously reported, even if the interval became longer. The observations by two observers were as follows. If the interval became longer, the continuity of movement disappears and the timing of emergence was too late, but the same object existed, and it was hidden under the screen, entrained and finally stripped off. It seems the entraining effect by Michotte and Thinès (1964), but the object is hidden and amodal (Michotte \& Burke, 1951).

To compare the favorite intervals, except timing criterion, the values were almost the same as in the preliminary experiment in the object-faster condition. However, in the screen-faster condition, as mentioned

${ }^{3} F_{(3,3)}=37.64, p<0.01 ; F_{(10,10)}=19.60, p<0.001 ; F_{(30,30)}=5.65, p<0.001 ; F_{(10,10)}=9.45, p<0.001 ; F_{(30,30)}=2.90, p<0.005$.

${ }^{4}$ For continuity, $F_{(1,44)}=17.40, p<0.001$ and, $F_{(1,44)}=15.16, p<0.001$; for timing, $F_{(1,44)}=6.26, p<0.05$ and, $F_{(1,44)}=3.79, p<$ 0.10 (significant tendency); for identity, $F_{(1,44)}=9.36, p<0.005, F_{(1,44)}=4.95, p<0.05, F_{(1,44)}=11.14, p<0.005, F_{(1,44)}=22.35, p$ $<0.001$ and $F_{(1,44)}=25.05, p<0.001$. 
above, the favorable intervals shifted into larger value. Thus, the perceptual impression of tunnel effect may not change, if the object as a screen moves slower than the hidden small object. But, if the screen moves faster than the small object, a kind of entraining effect appears and the impression of tunnel effect was different from the standing screen situation.

\section{Experiment 2}

\section{Purpose}

Continuously, it was confirmed if the favorable masking intervals changed according to the movement of screen. In the experiment 2 , the object and the screen faced each other, approached and passed. Thus, the opposite direction of movement in the face-to-face situation was examined (see Figure 6).

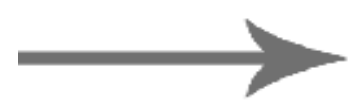

Figure 6. Situations examined in the experiment 2.

\section{Method}

Almost the same as in the experiment 1, but there were three levels in the factor of faster-object: same-speed, object-faster and screen-faster. In the same-speed condition, two objects moved in $100 \mathrm{~mm} / \mathrm{s}$. In other conditions, the speed of the faster was $66.7 \mathrm{~mm} / \mathrm{s}$ and the slower one was $33.3 \mathrm{~mm} / \mathrm{s}$. Thus, the relative speed was always $100 \mathrm{~mm} / \mathrm{s}$. Also, in experiment 2, there were dummy conditions to increase trials ${ }^{5}$.

\section{Results and Discussion}

Averaged numbers of satisfied trials for four criteria as a function of the interval condition were represented in Figures 7, 8 and 9, according to the faster-object conditions. In the same-speed condition, all of criteria had the best interval at the physical interval and, in the object-faster condition, all criteria but the continuity had the best interval in the same point. However, the greatest frequencies were generally smaller in the same-speed condition than in the object-faster condition. In the screen-faster condition, the favorable intervals appeared in the plus area of interval.

A three-way ANOVA with repeated measure was conducted and significantly revealed a main effect of the criteria, the interval, an interaction between the criteria and the interval, between the faster-object and the interval and an interaction among three factors ${ }^{6}$. To know the difference of favorable interval for each criterion,

\footnotetext{
${ }^{5}$ There were three conditions of the relative speed (mm/s) and screen's width (mm): (1) 50, 27; (2) 100, 54; and (3) $200,54$. These three conditions had three variations: the same-speed, the object-faster and the screen-faster. In total, there were nine dummy conditions.

${ }^{6} F_{(3,3)}=28.34, p<0.05 ; F_{(10,10)}=26.05, p<0.001 ; F_{(30,30)}=9.85, p<0.001 ; F_{(20,20)}=10.36, p<0.001 ; F_{(60,60)}=3.11, p<0.001$.
} 
the interaction among three factors was picked up. A simple-simple main effect revealed that for the continuity, at the $-15 \%$ interval, the difference among three faster-object conditions was significant and at the $+15 \%$ interval, the screen-faster condition got significantly higher score than other two conditions; for the timing, at the $+15 \%$ interval, the screen-faster condition got significantly higher score than other two conditions; for the permanence, at the $-15 \%$ interval, the screen-faster condition got significantly lower score than other two conditions; for the identity condition, at the $+60 \%$ interval and the $+75 \%$ interval, the screen-faster condition got significantly higher score than other two conditions ${ }^{7}$.

As in the Experiment 1, the favorable interval of the screen-faster condition tended to be at the plus area of interval. The impression of entraining in the identity criterion was also observed. Other report from two observers was about weaker continuity of movement in the same-speed condition. If the screen came for the object at the same speed and hid it, the impression of movement was easily cut out.

To compare the favorable interval in the preliminary experiment, the general tendency was the same as in the Experiment 1. If the screen moves slower than the small hidden object, the impression of tunnel effect does not change, comparing to the standard tunnel effect. However, if the screen moves faster than the small object, a kind of entraining effect appears and the impression of tunnel effect was different from the standing screen situation. If the both objects move with the same speed, the impression of tunnel effect is weakened.



Figure 7. Averaged number of frequencies of four criteria as a function of the interval in the same-speed condition: for the continuity, $\square$ for the timing, $\boldsymbol{\Delta}$ for the permanence and $\circ$ for the identity.

\footnotetext{
${ }^{7}$ For continuity, $F_{(2,88)}=17.29, p<0.001$ and $F_{(2,88)}=20.01, p<0.001$; for timing, $F_{(2,88)}=4.40, p<0.05$; for permanence, $F_{(2,88)}$ $=9.52, p<0.001$ and $F_{(2,88)}=3.17, p<0.05$; for identity, $F_{(2,88)}=5.83, p<0.001$ and $F_{(2,88)}=8.29, p<0.001$.
} 


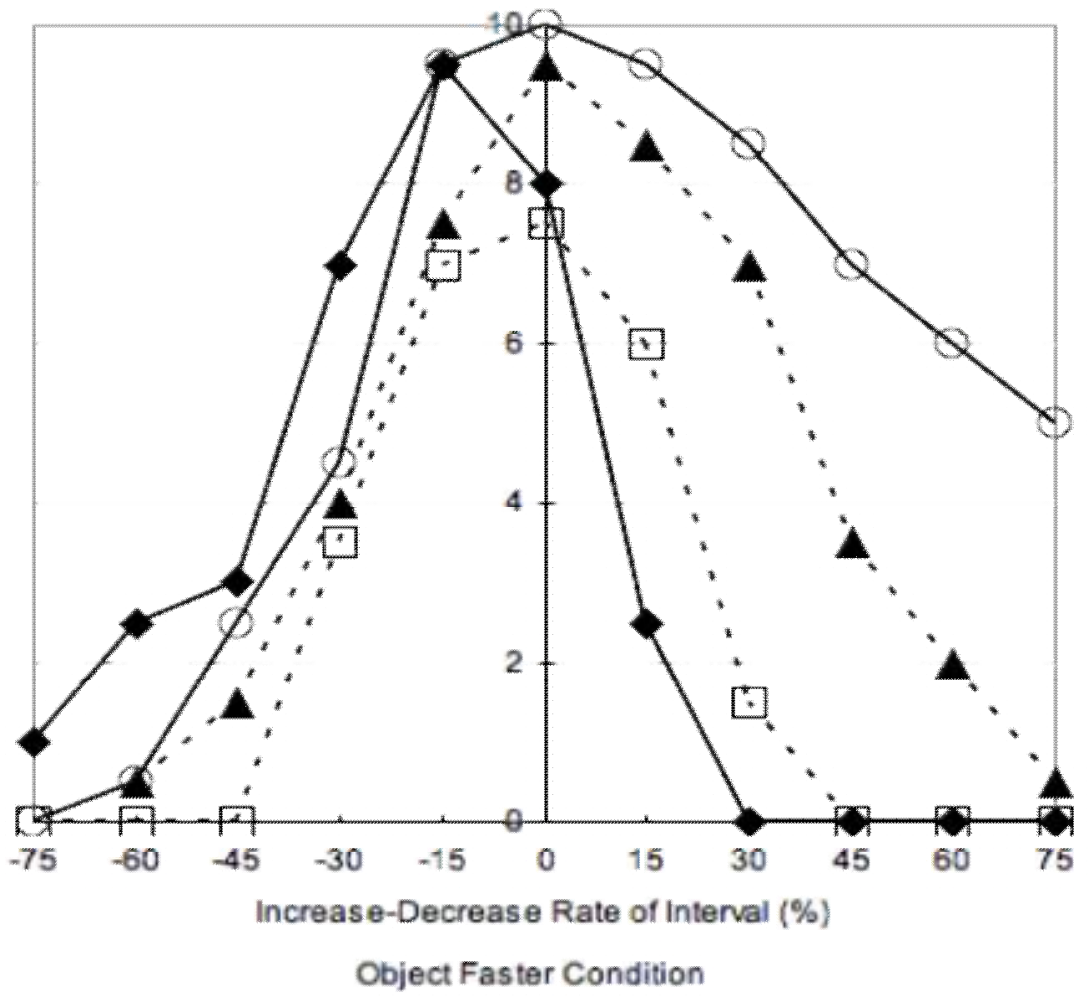

Figure 8. Averaged number of frequencies of four criteria as a function of the interval in the object-faster condition: $\checkmark$ for the continuity, $\square$ for the timing, $\boldsymbol{\Delta}$ for the permanence and $\circ$ for the identity.

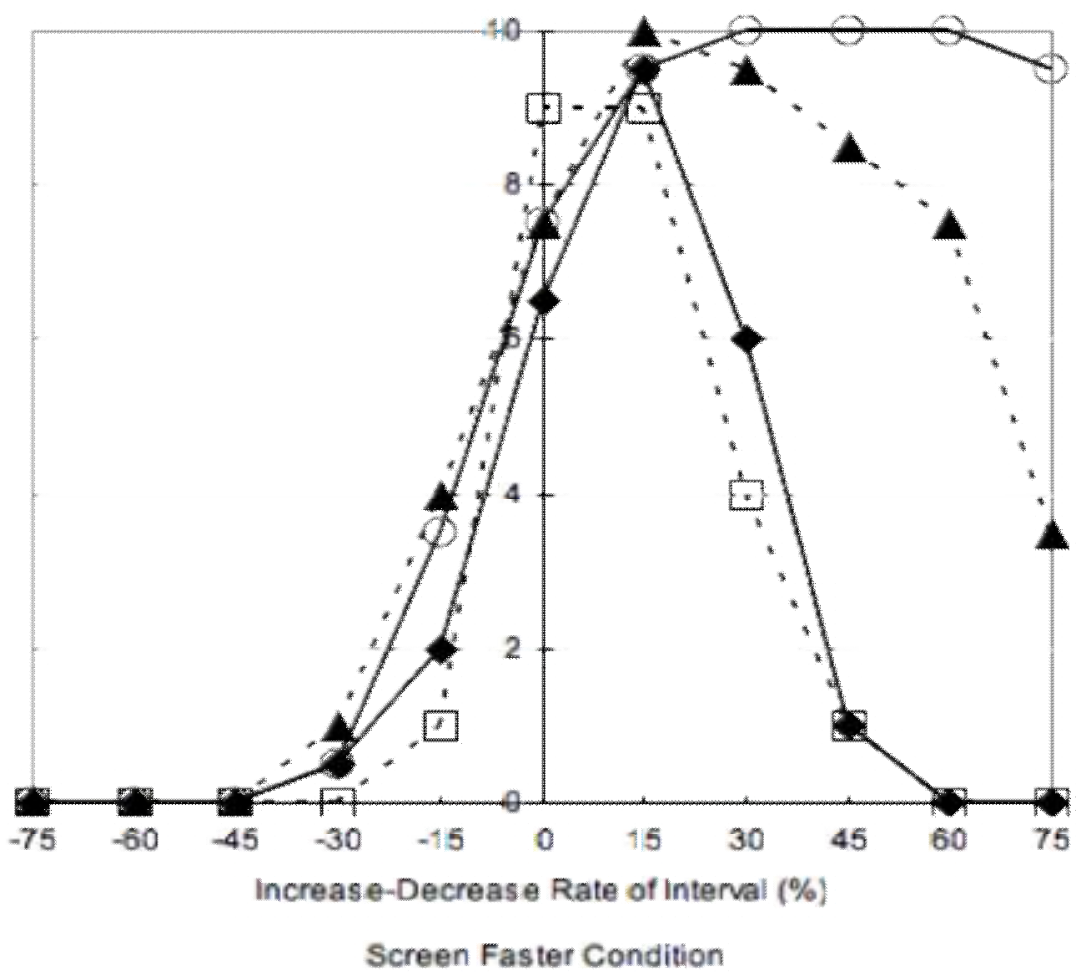

Figure 9. Averaged number of frequencies of four criteria as a function of the interval in the screen-faster condition: $\checkmark$ for the continuity, $\square$ for the timing, $\boldsymbol{\Delta}$ for the permanence and $\circ$ for the identity. 


\section{Conclusions}

The present study examined if the optimal interval to see the tunnel effect was always the same, despite of the movement of hiding object. Firstly, in the standard situation of tunnel effect, when the screen was standing and only the small object moved, the optimal interval varied according to the criterion and the speed of object. If the screen moved faster than the small object, the optimal interval became longer. In this situation, a kind of entraining effect was seen, even if the impression of movement under the screen disappeared. It was found that the effect was difficult to see if the screen came for the small object with the same speed. Thus, the tunnel effect cannot be explained only by the physical relationship between time and space. The relative size and speed, direction and the criterion that observers checked out affect to the emergence of tunnel effect.

Table 1 is a guideline about the occluding time to get the tunnel effect in a dynamic situation examined in the present study. The intervals are shown according to the physically-required one to pass each other for the object and the screen.

Table 1

Optimal Occluding Intervals to Get Certain Feature of Tunnel Effect When Both of the Object and the Screen Move: "PI" Means the Physically-Required Interval to Pass Each Other: " $<$ PI" Indicates the Interval Smaller Than the PI and "> PI" Is the Interval Bigger Than the PI

\begin{tabular}{llllll}
\hline & \multicolumn{2}{c}{ Same direction } & \multicolumn{2}{c}{ Face-to-face direction } \\
\cline { 2 - 6 } & Object faster & Screen faster & Same speed & Object faster & Screen faster \\
\hline To get smooth movement & $<$ PI & $>$ PI & - & $<$ PI & $>$ PI \\
Not to extinguish the object & $<$ PI & $>$ PI & - & $=$ PI & $>$ PI \\
\multirow{2}{*}{ To keep the timing of emergence } & $=$ PI & $=$ PI & $=$ PI & - & $=$ PI \\
& $<$ PI & & $=$ PI & $<$ PI & $>$ PI \\
\multirow{2}{*}{ To show the same object } & $=$ PI & $>$ PI & $>$ PI & $>$ PI & $>$ PI \\
& & & & & \\
\hline
\end{tabular}

\section{References}

Brandt, A. (1982). Phenomenal shrinkage and tunnel effect. Giornale italiano di Psicologia, 9, 323-334. Burke, L. (1952). On the tunnel effect. The Quarterly Journal of Experimental Psychology, 4, 121-138. Metzger, W. (1975). Laws of seeing (Kramer, Ed., Chapter XIII). Frankfurt: am Main. Kanizsa, G. (1991). Seeing and thinking. Bologna: Il Mulino.

von Kries, J. (1902). Ueber die im Netzhautcentrum fehlende Nachbilderscheinung und über die diesen Gegenstand betreffenden arbeiten von C. Hess. Zeischrift für Psychologie und Physiologie der Sinnesorgane, 29, 81-98.

Maruyama, K., \& Iwasaki, S. (1973). "Prospective perception” and the tunnel phenomenon. Tohoku Psychologica Folia, 32, 134-144.

Michotte, A., \& Burke, L. (1951). A new enigma of psychology of perception: "Amodal data" in the sensory experience. Proceedings and papers of the 13th International Congress of Psychology (pp. 179-180). Stockholm.

Michotte, A., Thinès, G., \& Crabbé, G. (1964). Amodal completions of perceptual structures. Louvain: Nauwelaerts.

Reynolds, H. N. J. (1968). Temporal estimation in the perception of occluded motion. Perceptual and Motor Skills, 26, 407-416.

Sampaio, A. C. (1943). Translation of objects as factor of phenomenal permanence. Louvain: Warmy.

Vezzani, S. (1998). Amodal completion in kinetic conditions. (Unpublished doctoral thesis, Università degli studi di Padova, Dipatimento di Psicologia generale).

Vicario, G. B., \& Kiritani, Y. (1999). Slow-motion tunnel effect: An inquiry into vertical organization of events. Gestalt Theory, 21, $100-121$. Wertheimer, M. (1912). Experimentelle studien über das sehen von bewegung. Zeitschrift für Psychologie, 61, 161-265.

Wundt, W. (1902). Characteristics of physiological psychology (pp. 582-583). Leipzig: Engelmann. 